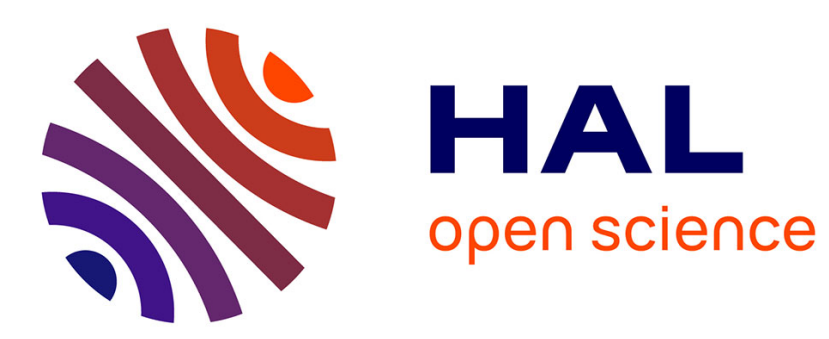

\title{
Le manifeste de Hesdin et ses réponses dans l'Auxerrois et le Mâconnais (1417-1435)
}

Benoît Léthenet

\section{To cite this version:}

Benoît Léthenet. Le manifeste de Hesdin et ses réponses dans l'Auxerrois et le Mâconnais (1417-1435).

Bulletin du Centre d'études médiévales d'Auxerre, 2010, 14, 10.4000/cem.11610 . halshs-03087401

\section{HAL Id: halshs-03087401 https://shs.hal.science/halshs-03087401}

Submitted on 23 Dec 2020

HAL is a multi-disciplinary open access archive for the deposit and dissemination of scientific research documents, whether they are published or not. The documents may come from teaching and research institutions in France or abroad, or from public or private research centers.
L'archive ouverte pluridisciplinaire $\mathbf{H A L}$, est destinée au dépôt et à la diffusion de documents scientifiques de niveau recherche, publiés ou non, émanant des établissements d'enseignement et de recherche français ou étrangers, des laboratoires publics ou privés. 


\section{Le manifeste de Hesdin et ses réponses dans l'Auxerrois et le Mâconnais (1417-1435)}

Le serment est un acte rituel qui institutionnalise la vie sociale dans ses aspects religieux, politiques et juridiques ${ }^{1}$. C'est un acte de la parole auquel sont liés des gestes, un acte qui fonde, garantit ou démontre. Le serment fait partie des rituels médiévaux qui fondent l'autorité normative et le lien social ${ }^{2}$. Par ailleurs, le serment a une fin et une efficacité qui lui sont propres puisqu'il comporte en lui-même un châtiment conditionnel en cas de parjure ${ }^{3}$. Les études sur le serment ont d'abord porté sur les aspects démocratiques du rituel. Il a été analysé comme un signe précurseur de nos démocraties modernes ${ }^{4}$. Puis, elles ont mis en avant la confiscation du pouvoir par l'oligarchie urbaine ${ }^{5}$, le rituel étant le moyen par lequel le groupe dominant met en scène sa légitimité et s'affiche comme le représentant de toute la communauté civique $^{6}$. Les études les plus récentes abordent la violence politique qui s'y exprime $^{7}$. Actuellement, les recherches menées par l'EA 3400 et l'UMR 7044 portent sur un projet commun inscrit au plan quadriennal intitulé Le rôle des serments dans les villes du Bas Moyen Âge (axe : Liberté et contrainte). Le projet espère notamment combler le problème du rapport entre l'oral et l'écrit. Lors de recherches dans les archives départementales de la Côte d'Or, menées dans le cadre de notre travail de doctorat, nous avons consulté la liasse B 11895 qui contient une vingtaine de lettres d'adhésion à Jean sans Peur (1417). Ces lettres d'adhésion ont donné lieu à des serments qui prennent la forme de l'hommage.

Dans l'action politique, le serment est l'un des processus qui peuvent politiser les acteurs, définir un espace public et provoquer une interaction au sein de cet espace, entre les princes et les sujets ${ }^{8}$. Jürgen Habermas décrit le pouvoir politique au Moyen Âge comme un pouvoir monolithique, imposé par le haut sans discussion, ni retour critique possible, de la part de bourgeois privés d'agir politique ${ }^{9}$. Depuis le livre de Jean-Claude Perrot, Genèse d'une ville moderne : Caen au XVIIIe siècle (1975), les historiens dénoncent la conception d'un « espace vide et comme indifférent à l'action ${ }^{10} »$. Ils invitent à se méfier du schéma narratif dans lequel il y a un âge d'or, celui des Lumières, où s'épanouissent des processus d'apprentissage et de discussion, creusets de la découverte de la politique. Au Moyen Âge ${ }^{11}$, le peuple n'est pas dans une posture passive face à l'espace public où se déploie le pouvoir. La concurrence acharnée et violente que se livrent les princes, les incite à rechercher ressources et alliés auprès des villes. Cette concurrence oblige au dialogue avec les élites urbaines et appelle les princes à mieux investir l'espace public. Dégager cet espace et le défendre implique la nécessaire politisation des gens des villes ainsi que la naissance d'une opinion publique ${ }^{12}$. L'espace public devient alors le lieu du débat politique et du débat d'idées ${ }^{13}$.

Les ordonnances ${ }^{14}$ de police des autorités urbaines témoignent du souci constant de délimiter par le droit un espace soumis à un régime particulier. Ces statuts, qui ont comme fondement le bien commun, nous offrent des «récits d'espace $^{15}$ » dans lesquels existe un jeu subtil entre les dimensions du public, du semi-public et finalement du privé ou du secret ${ }^{16}$. Ces sous-espaces, permanents ou occasionnels, peuvent conserver une marge d'autonomie importante. Il est impossible de limiter l'espace public aux lieux juridiquement définis. Déterminer par le droit, il est surtout qualifié par l'usage ${ }^{17}$. Le renforcement des moyens de communication (routes, messageries, pratique du vidimus) participe à la politisation des gens des villes. La cité offre un cadre matériel qui n'est pas entièrement soumis à l'emprise du prince. Elle s'affirme comme le premier metteur en scène de toute communication politique ritualisée au cœur de l'espace festif ${ }^{18}$ créé à cette occasion. La mise en spectacle du rite ${ }^{19}$ tient compte des contingences liées au décor urbain ${ }^{20}$. Les lieux de la ville guident et contraignent les pratiques des acteurs ${ }^{21}$. En tant que scène d'interaction sociale, la domination de cet espace public constitue pour le prince un relais important entre lui et ses sujets bien que la pluralité des influences s'exprime notamment dans le cas extrême des révoltes et des désaccords ${ }^{22}$. Il n'est 
pas non plus impossible que de «vraies » manifestations publiques puissent emprunter d'autres canaux comme ceux de l'image, du symbole ou de la subversion. L'espace public peut donc être abordé comme un lieu de parole ${ }^{23}$ et de mémoire façonné par les affaires communes. Requérir un serment de fidélité politise de fait les bourgeois, les place au cœur de l'espace public défini comme lieu de parole et de mémoire, et permet au prince d'investir cet espace.

Les chercheurs ne se sont pas emparés d'un thème pourtant très prometteur. Les premiers recueils de pièces «curieuses ${ }^{24}$ » de la région ne mentionnent pas les lettres de Hesdin. L'original en français est édité intégralement par Dom Plancher ${ }^{25}$ puis, au siècle suivant, par Barante $^{26}$. Ils utilisent le document comme une preuve mise au service de l'histoire générale qu'ils construisent, sans percevoir que le manifeste (qui questionne le récepteur) est une « prise » sur le récepteur (qui répond au manifeste). Parallèlement, l'original en latin est resté totalement oublié. Le travail d'érudition et d'édition de Marcel Canat ne donne lieu à aucun développement sur le sujet. C'est dans 1'histoire des villes ${ }^{27}$ et des provinces ${ }^{28}$, entamée au XIXe siècle, que nous avons rencontré les premières mentions des réponses. Celles de Vézelay, Saint-Bris et Brinon ont été reproduites intégralement mais toujours à l'appui du récit. La réponse de Charlieu, très écourtée, donne lieu à une analyse succincte.

L'historiographie locale nous donne davantage à voir une chronique des libertés bourgeoises ${ }^{29}$ que le rapport de force qui s'instaure entre l'émetteur et les récepteurs du manifeste de Hesdin. La recherche universitaire ne méconnaît pas le document ${ }^{30}$ mais ne s'est pas saisie, spécifiquement, de ce dossier. Le processus de diffusion des lettres, l'introduction de la scripturalité dans l'oralité, le jeu de question/réponse comme acte de domination n'ont pas encore fait l'objet de recherches.

Nous voulons, ici, donner une première étude qui sera plus largement développée dans le cadre d'une thèse de doctorat ${ }^{31}$, à l'université de Strasbourg, sous la direction de Georges Bischoff.

\section{Contexte}

Dés la fin du XIVe siècle des tensions se marquent entre Louis d'Orléans, frère de Charles VI (1380-1422), et son oncle Philippe le Hardi, duc de Bourgogne. La personne du roi, soumise à des «fièvres », est affaiblie et ballottée par les évènements. Les défaillances mentales du roi deviennent évidentes après l'épisode de la Forêt du Mans, en août 1392, lors de sa chevauchée vers la Bretagne. Les ducs se disputent pour le décharger du poids du gouvernement. Jean de Berry et Philippe le Hardi se montrent solidaires face à leur nouvel adversaire : Louis duc de Touraine et d'Orléans. La rivalité des princes porte essentiellement sur l'accès au trésor royal qui leur permet de financer leur vie de cour, leur politique personnelle et leur clientèle ${ }^{32}$. Les rivalités s'exacerbent après la mort, le 27 avril 1404, de Philippe le Hardi. Jean sans Peur, son fils qui lui succède, ne bénéficie plus de la même manne financière. A la fin de son principat, entre 1396 et 1404, Philippe percevait annuellement 176.500 1. t. du roi. A sa mort, son fils ne perçoit plus rien. Il lui faut attendre mars 1405, date de son avènement aux comtés de Flandres, d'Artois et de Bourgogne pour connaître un accroissement de ses revenus. Dans le même temps, Louis d'Orléans voit ses positions se renforcer. Jean sans Peur en vient à faire assassiner son rival, le 23 novembre 1407, rue Vieille du Temple à Paris. La discorde des princes se mue en guerre civile.

Guerres, alliances et paix fourrées se succèdent entre les Bourguignons et les Orléans dont Bernard d'Armagnac et son gendre Charles, fils de Louis d'Orléans, se font les animateurs. Charles peut compter sur le soutien du duc de Berry, son grand-oncle, sur celui de ses cousins 
Anjou et Bourbon, ainsi que sur l'appui du duc de Bretagne et celui de sa tante Isabeau de Bavière, reine de France. Les princes hostiles au duc de Bourgogne forment en avril 1410 la ligue de Gien ${ }^{33}$ alors que Jean sans Peur rallie à sa cause le roi de Navarre, Charles le Noble, fils de Charles le Mauvais ${ }^{34}$. "Bourguignons » et «Armagnacs» s'affrontent pour la domination du gouvernement royal, pour le contrôle du dauphin et l'extension de leur espace d'influence. Les villes, au cœur des exactions et des négociations, sont régulièrement sommées de prendre parti et doivent négocier avec les protagonistes aux mieux de leurs intérêts.

La victoire anglaise d'Azincourt, le 25 octobre 1415, débarrasse le duc de Bourgogne d'un certain nombre de ses adversaires, morts au combat ou faits prisonniers (principalement les ducs d'Orléans et de Bourbon). Le parti bourguignon a subi lui aussi des pertes notables. Les frères du duc de Bourgogne, Antoine de Brabant et Philippe de Nevers, comptent parmi les tués. L'espace politique ainsi vidé permet au duc de Berry et au dauphin Louis de Guyenne, plus modérés, de jouer une autre partition. Cependant, le dauphin Louis meurt en décembre 1415 et le duc de Berry en juin $1416^{35}$. Jean, le nouveau dauphin, disparaît à Compiègne le 5 avril 1417 peu de temps avant Louis d'Anjou qui s'éteint à Angers le 29 avril $^{36}$. Par la force des choses c'est un personnel nouveau qui entoure le roi et Charles, le jeune dauphin. Le panetier du roi Pierre de Fénin porte un regard juste sur la nouvelle situation que créent folie, captures et décès :

«Alors gouvernoient le roy et le dauphin le comte d'Armagnac, le seigneur de Barbasan et Tanneguy Du Chastel [...] et tous gens qui estoient estrangers pour la plus grande partie ; lesquels pour ce ne vouloient point que ledit duc Jean fut bien d'accord avec le roy et avec le dauphin, pour ce qu'ils sçavoient bien que si le duc Jean y estoit, il leur osteroit le gouvernement qu'ils avoient dans le royaume ${ }^{37}$.»

Le duc de Bourgogne reste le seul prince du sang apte à faire valoir son rang pour prendre la direction des affaires aux côtés de Charles VI et du dauphin Charles. Il fait publier des lettres patentes dans lesquelles il explique les motifs qui le poussent à agir. Le Languedoc est gagné par les Bourguignons et de nombreuses villes font défection en sa faveur. A partir de 1418, le dauphin Charles prend la tête du parti armagnac qui devient le " parti dauphinois ». L'entrée des Bourguignons à Paris, en mai 1418, s'accompagne du massacre de leurs opposants au cours duquel Bernard d'Armagnac est tué. Charles s'installe à Bourges. Jean sans Peur qui a délivré la reine de son exil à Tours forme un nouveau gouvernement à Troyes. Comme une réponse au meurtre de 1407, le duc de Bourgogne est lui-même assassiné, le 10 septembre 1419, sur le pont de Montereau par les hommes du dauphin. Charles VI, finalement passé sous l'influence bourguignonne, déshérite son fils rendu responsable de l'assassinat du duc de Bourgogne. Le traité de Troyes fait passer à la dynastie Lancastrienne la couronne de France (21 mai 1420). Opérations militaires et traités de paix se succèdent. C'est généralement au congrès d'Arras, en 1435, que les historiens arrêtent leurs récits de la guerre civile.

\section{Un corpus homogène}

Dans les rivalités exacerbées et les conflits qui opposent les princes, les villes sont sommées de prendre parti ou de réitérer leur fidélité. Lors de la tentative de conquête du pouvoir par le duc de Bourgogne ${ }^{38}$, en avril 1417, le clergé et les habitants de Mâcon envoient le 5 septembre ${ }^{39}$ des lettres de serment en réponse aux lettres de Hesdin datées du 25 avril $^{40}$. Dans ses lettres, Jean sans Peur emploie les références fondamentales du discours de paix qui montrent qu'il est un vrai prince de paix ${ }^{41}$. Cette posture repose sur la déploration des 
malheurs de la guerre, le refus de verser le sang chrétien, les tentatives de paix répétées et la mise en œuvre constante de tous les moyens pour y parvenir, enfin la volonté d'aller jusqu'au sacrifice du sien et de soi. Les Mâconnais, contraints par l'envoi de lettres et de proches de l'entourage ducal, ont "promis, juré et sur ce baillié lettres [...] de enhuy emploier a lui aydier de tout leur povoir sans y voloir riens espargnier a sa dicte entencion et son propos exequter $^{42} \gg$. Le serment de 1417 assure une nouvelle domination, celle de la Bourgogne, imposée par l'élite mâconnaise ${ }^{43}$ aux habitants de la cité. Les archives départementales de la Côte d'Or conservent une vingtaine de lettres de serment semblables à celles de Mâcon ${ }^{44}$. Ces lettres sont des chartes ${ }^{45}$. Elles ont comme objet juridique l'adhésion accordée par les autorités municipales et sont validées par le sceau appendu au bas du document. Leur répartition géographique et chronologique permet de distinguer trois groupes [Fig.1] :

1) Le premier document est un acte produit au nord de Dijon, le 19 juillet 1417. Il est le plus précoce de la série mais reste isolé géographiquement et chronologiquement. Il s'agit d'une certification réalisée par le bailli de Langres pour le compte du lieutenant du bailli de Chaumont, en l'absence de ce dernier. Le sceau du bailliage de Langres est appendu au bas du document qui contient une copie de la déclaration d'adhésion des bourgeois de Chaumont.

2) Les six actes suivants forment un groupe homogène. Ils proviennent tous du Mâconnais. Les trois premiers sont réalisés à la frontière du Chalonnais et le long de l'axe de la Saône. Ils proviennent de Saint-Gengoux (25 août), de Tournus (28 août) et de Mâcon (5 septembre). Les lettres d'adhésion de Marcigny (28 septembre), BoisSainte-Marie (30 septembre) et Charlieu (14 octobre) diffèrent par leur date plus tardive et par leur localisation à la frontière avec le Beaujolais. Ce groupe permet le mieux de saisir la diffusion spatiale et chronologique du mouvement d'adhésion.

3) Les dernières lettres proviennent de l'Auxerrois. Il s'agit des seigneurs ou des bourgs ruraux de Chablis (3 octobre), Sacy, Villefranche, Charny (6 oct.), Villiers-SaintBenoît, Chêne Arnoult (7 oct.), Brinon (8 oct.), Mailly-le-Château (10 oct.), SaintBris, Vézelay et Vermenton (14 oct.). On est frappé de n'y trouver aucune localité d'importance. Cet aspect est confirmé par la taille et la mise en forme des actes, plus minces et moins élégants que les documents mâconnais.

Le duc s'assure, à différentes échelles, la maîtrise du territoire et celle des principales places fortes de la région, avec une finesse plus grande que nous laissent percevoir les chroniqueurs du XVe siècle. Ce qui lui permet la couverture militaire de ses frontières au sud ${ }^{46}$. Jean sans Peur, en requérant les serments de fidélité du Mâconnais et de l'Auxerrois, anticipe les propos de Charles VII : «nous avons envoyé et chacun jour envoyons, vers le pays de Bourgongne, gens à souffisance pour donner assez a faire au dit duc de Bourgongne a garder son pays ; et tellement y avons porveu et pensons encore plus porvoir en brief, que besoin luy sera de soy et tous les siens occuper a la garde de son dit pais, sans avoir espérance de soy autre part employer $[\ldots]^{47} \gg$. Les lettres sont des documents de gestion du territoire à des fins politiques. Elles répondent aux sollicitations de Jean sans Peur. Les lettres de Hesdin ont été « exhibées et leues publiquement oudit lieu de Mascon ». Exhiber les lettres permet d'en montrer le support, les dimensions, la mise en page, les moyens de validation toutes choses utiles afin de «bien a plain acertenés et veritablement informés » les destinataires sur l'origine des lettres. Ces garanties donnent l'information comme vraie, laquelle reste l'enjeu de nombreux conflits. Rappelons que les lettres placardées à Mâcon, en juillet 1417, par des partisans probourguignons, étaient des copies non valides sans scellement ni signature des lettres de Hesdin. Le 16 juillet, lorsque le chevaucheur envoyé par Marguerite de Bavière arrive à Mâcon, il présente aux bourgeois une nouvelle copie «en papier non signée ${ }^{48}$ » qui est 
aussitôt contestée. La lecture publique, n'intervient qu'une fois l'authenticité du vidimus établie et constatée par tous. De même, il est peu probable que des originaux soient parvenus à Marcigny, Bois-Sainte-Marie et Charlieu, bien que les réponses mentionnent la « vision des lectres patentes ${ }^{49}$ ». Ce sont des copies vidimées ${ }^{50}$, expédiées depuis Mâcon, collationnées à un document «de seur estat » lui-même vidimé à Dijon $^{51}$. Ce procédé pose la question de la production en série de certains documents avant l'invention de l'imprimerie ${ }^{52}$. Il pose aussi la question de la langue car l'ensemble des documents sont en français. L'original latin $^{53}$ des lettres de Hesdin conservé aux Archives à Dijon ne semble pas avoir été repris et reste un document unique ${ }^{54}$. L'écrit en français sert bien, ici, à administrer un territoire ${ }^{55}$. Cependant, c'est la réponse qui est faite à la demande de Marguerite de Bavière, qui enjoint aux bourgeois de faire connaître le contenu des lettres «par manére de publicacion ou autrement le mieux que vous povés notiffier et fere savoir es lieux les plus notables et prochains de vous en telle manére qu'il puisse venir a la congnissance des gens d'eglise nobles et bourgeois de vouz marches voysines. ${ }^{56} \gg$ L'usage de la copie apparaît comme une procédure à part entière et une alternative au cri d'information ${ }^{57}$. Elle permet en tout cas de se reporter commodément au texte, qui s'applique à rendre l'autorité de l'original, surtout lorsque la distance ne permet pas de prendre connaissance du document premier. Enfin, l'un des critères d'authentification est à rechercher dans le système de l'archivage des actes, sous la responsabilité du procureursyndic de la cité. Le document dijonnais et une copie ont été déposés dans l'arche à la chapelle Saint-Nizier de Mâcon. En cas de contestation, il suffit de s'y reporter. Les deux actes, par ce moyen de conservation, sont parvenus jusqu'à nous.

\section{Le rituel du serment}

Le plébiscite recherché par Jean sans Peur peut déboucher sur un serment, lequel ne paraît pas avoir été systématique. En effet, il n'est pas évident que les expressions «promectons » et «jurons » recouvrent exactement la même réalité. Si cela n'est pas le cas, ce ne sont que $36,8 \%$ des localités qui prêtent effectivement serment au duc de Bourgogne.

\begin{tabular}{|l|c|}
\hline Expressions de l'adhésion & citations \\
\hline « ont accordé et permis...» & 1 \\
\hline «promectons... & 11 \\
\hline «promectons et jurons... $»$ & 4 \\
\hline $\begin{array}{l}\text { «leur foi pour ce bailler corporelement en notre main... } \\
\text { «la foi et serment de nos corps donnés corporelement es mains... » } \\
\text { «faire promesse et serment en la main...» }\end{array}$ & 3 \\
\hline
\end{tabular}

Le rituel du serment est le temps de la parole masculine et du geste viril. Il se décompose en quatre étapes :

- La première étape consiste à rassembler la communauté des habitants concernés par le rituel. A Mâcon, elle comprend d'ordinaire un représentant du bailli et du prévôt, le doyen ou un représentant du chapitre de Saint-Pierre, les échevins et plusieurs bourgeois ${ }^{58}$. Il s'agit, lorsque les noms nous sont connus, des maîtres des métiers. Dans les localités de l'Auxerrois la communauté s'affirme, dans la majorité des cas, comme la personne d'autorité en milieu rural («Nous, les habitants de ..., certiffions »). Les assemblées d'habitants, sont les auteurs des actes d'adhésion. On compte 41 personnes à Brinon, 65 individus à Vermenton et 95 à Saint-Bris qui se déclarent «la plus grant et saine partie des manans et habitants ». On peut estimer que la quasi-totalité des chefs de feux sont réunis. Plusieurs familles sont représentées par deux voire trois de leurs membres. Ce qui fait à Saint-Bris une cinquantaine de familles liées par des intérêts divers. Ce sont des maîtres des métiers, des artisans ou des paysans. 
- La seconde étape consiste à lire à haute voix les lettres expliquant les motifs du serment. Dans le cas de l'adhésion des habitants de Saint-Bris, les lettres « ont esté monstrées et exhibées » par l'envoyé ducal. Jacques de Courtiambles, seigneur de Commarin, est conseiller et chambellan de l'hôtel du roi et du duc de Bourgogne. Cette double appartenance facilite l'adhésion des bourgeois de Saint-Bris. L'officier expose la bonne volonté de son maître et son intention de réformer le gouvernement du royaume. Les bourgeois voient les lettres, mais il n'est pas dit, à l'inverse du cas mâconnais, qu'ils les ont lues eux-mêmes. La publication faite par Jacques de Courtiambles est vraisemblablement une publication populaire abrégée du texte de Jean sans Peur. Il semble que la publication ne tire que la morale du manifeste de Hesdin ( «la bonne voulenté » du duc / « le present bien du roy » / «la paix » / « la tranquillité »). Si l'acte d'adhésion de la communauté des habitants de Saint-Bris nous a effectivement conservé les points forts de la publication orale à laquelle elle assiste, on doit la limiter à deux points principaux : les malheurs du royaume et l'engagement ducal. Ainsi, à côté de publications savantes comme à Mâcon, où les textes sont lus in extenso, existe une publication populaire, c'est-à-dire un résumé bref des lettres de Hesdin. Les premiers informés sont donc les bourgeois des villes et des villages, ce qui pose la question du pouvoir politique des élites en milieu rural et de la circulation des idées politiques parmi les élites paysannes ${ }^{59}$.

- Une fois les «lectres veues » les bourgeois de Saint-Bris déclarent : «nous a esté par ledit maitre de Commarien requis nostre entencion et voulenté en icelles ». La prise de parole marque une troisième étape et nous conduit au cœur de l'interaction entre les acteurs de la publication. Jacques de Courtiambles est un représentant de la haute noblesse de la région, conseillant la duchesse sur les questions militaires. Il manifeste, par sa présence, la puissance de la Bourgogne et rappelle sa proximité. Les bourgeois «acertenés et informés veritablement » par le jeu du vidimus, de la lecture publique et des relations qui leur sont faites de l'adhésion des localités voisines saisissent les enjeux du ralliement. Ils le discutent ( «en regart et deliberacion éz chouses dessusdites »). La suite sonne comme un aveu de faiblesse, au mieux d'obéissance, ou les deux. L'adhésion s'opère « selon ce que les bourgois manens et habitans en la ville et cité d'Aucerre l'ont juré juré [sic] et promis et que contenu est és lettres du serment et aliences faiz par les gens d'eglise bourgois habitans et manens en la dite ville et cité d'Aucerre.» Plus que la participation des bourgeois au bon gouvernement que le duc de Bourgogne se propose de rétablir, c'est leur obéissance qui s'exprime dans la cérémonie du serment.

- La quatrième étape est celle d'un serment qui prend la forme de l'hommage. A Mâcon la lettre d'adhésion et sa confirmation par la duchesse de Bourgogne anticipent un acte juridique qui n'intervient que le 17 septembre. Le chancelier de Bourgogne, Jean de Saulx, revient à Mâcon pour recevoir le serment de fidélité. Il vient "prendre obeissance ${ }^{60}$ »après avoir préalablement fourni de solides garanties financières et militaires. La lettre d'adhésion est une déclaration d'intention que complète une cérémonie ultérieure. Dans le cas de Brinon les lettres d'adhésion ajoutent, en l'insérant dans le corps du document, le «texte » d'une lettre qui donne à un fondé de pouvoirs la charge de prêter le serment demandé. Le fondé de pouvoirs déclare que les bourgeois s'engagent à :

« faire promesses et sermens es mains de noble et puissant seigneur monseigneur Jaques de Courtiambles [...] gouverneur ordonné ou pais d'Aucerrois ou d'autre qui ont de lui puissance et que lesdiz habitans et chascun d'eulx se voulent et consantent eulx emploier selon leur possibilité au bien du roy notre seigneur de son royaulme et de mon dit seigneur de Bourgoigne et de la chose publique selon la forme et teneur des lettres envoyés et baillées audit monseigneur Jaques par lesdiz habitans. Et aussi comme autrefoiz l'on juré et promis es mains de monseigneur le conte de Joigny ${ }^{61}$. Et generaulement de fere autant des choses dessus 
dites et de leurs deppendances comme ilz conjointement et divisiement faire pourroient se presens y estoient tous en leurs personnes ja soit ce que la chose requist mandement especial promectans yceulx constituans par leurs foiz pour ce bailler corporelement en notre main et sur l'obligation de tous leurs biens meubles et immeubles presens et advenir et tenir et avoir ferme estable et agreable a tous jours sanz rappel tout ce qui par leurs diz procureux et l'un l'autre ou pour l'un d'eulx sera fait plaidié procuré besoigné et autrement ordonné des choses dessusdites et de leurs deppendances. Et paier la chose advigée se mestier est. »

Cet engagement est le cour de l'hommage. Il est conforme à la teneur des lettres d'adhésion. Les communautés se déclarent volontairement en faveur de Jean sans Peur («déclarons entièrement » dans $31,5 \%$ des cas, «adhérons » pour 52,6\% des chartes) et se placent dans la main du représentant ducal. L'absence de toutes mentions du parjure incite à reconnaître et un affaiblissement du caractère sacré du serment, et une forme contractuelle de la cérémonie proche de ce qu'est l'hommage.

\section{Les lettres d'adhésion}

La manifestation de l'autorité de ces assemblées passe par la commande de l'acte écrit auprès d'une juridiction gracieuse. Ces actes «commandés » par l'échevinage rural ou urbain sont faits dans des parchemins d'une qualité relative et de petite dimension [Fig.2]. Leur forme est très allongée. Les actes dénotent d'un souci de la présentation bien que les réglures soient encore visibles sur plusieurs documents. La justification du texte est parfaitement respectée à gauche mais l'alignement, à droite, a souvent pour limite le bord du parchemin. Une lettrine, de petite dimension, parfois délicate, est présente à la première ligne. Le module de base de l'écriture est de 1 à $2 \mathrm{~mm}$ alors que les interlignes varient de 4 à $7 \mathrm{~mm}$. L'écriture cursive se révèle soignée et régulière. Les abréviations sont nombreuses, 31 dans l'acte d'adhésion de Chablis, soit entre deux et trois par ligne. Le système abréviatif varie peu entre les actes et reste cohérent à l'intérieur d'un même document. Le scribe écrit et abrège les mêmes mots de la même manière. Le français est bien maîtrisé. Les documents présentent des marges généreuses à gauche, jusqu'à $57 \mathrm{~mm}$, et en haut, jusqu'à $25 \mathrm{~mm}$, compte tenu de la taille réduite des actes. Le repli inférieur peut atteindre $30 \mathrm{~mm}$. L'emprise de l'écrit est parfois extrême, $76 \%$ de la surface du parchemin de Brinon, mais la moyenne reste de 55\%. Les juridictions gracieuses affichent leur puissance en gaspillant une superficie aussi importante que les documents ducaux (56\%). Toutefois, par leur aspect général, leurs dimensions, les documents traduisent une rapidité extrême d'exécution et un manque de solennité.

Le discours diplomatique et les propos des lettres ont évolué depuis les lettres de Chaumont. Ils sont homogènes et même standardisés. Les aspects dépassés sont rapidement abandonnés y compris dans le groupe de lettres du Mâconnais. On ne fait plus mentions, comme cela est le cas dans la lettre de Saint-Gengoux, de la querelle qui oppose Jean sans Peur au roi de Sicile. Les particularités locales et techniques du ralliement sont gommées. L'engagement «jusqu'à la mort » au côté du duc est passé sous silence. Les indications portant sur l'absence des plus hautes autorités religieuses ${ }^{62}$ et laïques ${ }^{63}$ ne sont pas reprises. On peut noter la concomitance entre ces absences et le concile qui se tient à Constance (1414-1417). Les lettres du groupe de l'Auxerrois sont interchangeables. Une lettre type, comme celle de Chablis [Fig.3], se compose d'une suscription (« Nous - certiffions », 1.1) suivit d'une adresse générale («a tous - verront », 1.1) sans salut. La communauté rurale s'affirme d'emblée comme le suscripteur de $l^{\prime}$ acte $^{64}$. Le «texte » proprement dit ne comporte ni préambule ni notification mais débute par la narratio («Que par noble homme - notre entencion et volonté sur icelles », 1.1-7). Le dispositif de l'acte est clair et sans équivoque, il annonce l'intention des habitants d'adhérer au projet de réforme de Jean sans Peur («A quoy nous avons respondu - Bourgoigne », 1.7-8). Une clause de garantie complète le dispositif («Et jurons - publique », 1.9-10») 
immédiatement suivie par l'annonce expresse des signes de validation ( «En tesmoing présentes », 1.10-11). L'eschatocole comprend la date («Donné - dix sept», 1.11-12) et, plus rarement, une mention hors teneur fait figurer le nom du scribe. Le texte se veut concis et efficace, repris d'un formulaire type, élaboré au gré des réponses données entre juillet et octobre, qui valorise le consensus autour des réformes de Jean sans Peur. Cette réponse "prête à emporter» explique l'extraordinaire rapidité de la tournée de Jacques de Courtiambles dans la région. Un exemplaire de la réponse à donner, en plus du vidimus des lettres de Hesdin, devait circuler.

\section{Négligences ou résistances?}

En dépit de la mention des clauses de validation, les lettres de Mailly-le-Château, Vézelay et Vermenton n'ont pas été scellées. A Vézelay c'est le sceau de la juridiction séculière de l'abbé qui est absent, alors qu'à Mailly-le-Château et Vermenton c'est le sceau de la prévôté. Ces documents ruraux témoignent d'un affaiblissement du recours à cette forme de corroboration alors que le seing notarial tend à se substituer au sceau.

Dans le cas de Vermenton, les bourgeois ont commandé des lettres intitulées au nom du prévôt. C'est par sa volonté que se sont rassemblés les bourgeois. Le notaire a mis le texte au propre reprenant le modèle ( ?) donné par Jacques de Courtiambles. Une fois achevée la mise au net, l'acte est ensuite remis aux bénéficiaires par le notaire, qui a signé le document de sa main. La charge incombe aux bourgeois de le faire sceller. La rapidité d'exécution peut expliquer l'absence du sceau. Pourquoi allonger les délais puisque l'assemblée des Vermentonnais a tranché en faveur du duc. L'important est que l'acte soit rédigé et expédié. Le scellement du document nécessite de mandater et de dédommager un clerc auquel est confiée la charge de poursuivre l'affaire. Il convient aussi de payer l'émolument du sceau et son poids de cire. La dépense s'avère lourde, à Mâcon elle s'élève à 101 . t. par an ${ }^{65}$ au début du XVe siècle. Les échevins ont recours à la chancellerie de la prévôté une quarantaine de fois par an et à ces dépenses s'ajoutent les sommes versées à l'officialité de l'évêque. Acte de pure administration bourgeoise, sans réelle valeur solennelle, le scellement du document de Vermenton échappe au contrôle de la chancellerie de la prévôté. Il faut croire que la preuve de l'adhésion réside davantage dans le témoignage de tous ceux qui ont présidé à l'acte de soumission que dans le scellement du document. Vermenton, comme Saint-Bris ou Brinon, recourt à l'écrit authentique difficilement contestable. Les témoins vermentonnais, au total 65 individus présents «en leur personne », certifient l'action engagée et leur témoignage offre une garantie suffisante. Le cas échéant, si l'acte est contesté lors de son enregistrement, le prévôt le confirme par son sceau.

Le document provenant de Mailly-le-Château n'est pas scellé pour des raisons différentes. L'acte commandé par les habitants affirme qu'ils ont «requis et obtenu le seel de la dicte prevosté ». Ce document a été rédigé par deux notaires, deux frères, qui ont signé l'acte de leur seing notarial. La signature des notaires a remplacé le sceau du prévôt rendu moins indispensable puisque le rituel du serment a déjà eu lieu - contrairement à Mâcon. L'absence du sceau est dans ce cas une simple négligence. Toutefois, il est possible que cet oubli soit lié à la volonté d'échapper au contrôle gênant des membres de la chancellerie, ou de la bourgeoisie, opposés au ralliement ${ }^{66}$ à la Bourgogne.

Il est probable que dans le cas de Vézelay cet oubli soit tout aussi coupable. Un acte non scellé est réputé suspect, ce qui ne peut pas échapper aux contemporains. L'abbé de Vézelay, Pierre II de Modon ${ }^{67}$, n'authentifie pas l'acte et il n'en est pas le suscripteur. Ce sont les habitants qui déclarent : «Nous, les habitans de Verzelay, certifions [...] ». Aussi, sans une liste de témoins, sans la signature du notaire, alors que le rituel du serment n'a pas eu lieu, l'engagement des Vézeliens n'est garanti que sur un parchemin non authentifié. L'absence du sceau est bien le signe d'une résistance. Les religieux sont favorables à la légitimité royale 
comme le montre l'exemple de l'abbé Alexandre. Il exhorte les Vézeliens à quitter la ligue anglo-bourguignonne et contribue personnellement au rapprochement entre Charles VII et Philippe le Bon.

La marge de manœuvre des communautés rurales et des seigneurs locaux est bien mince. Il semble cependant qu'une forme de contestation larvée ait trouvé à s'exprimer. Une seconde manière de résister porte sur la modification, a posteriori, du texte de la lettre d'adhésion. Dans le document provenant de Chablis, la lettre mise au propre a été corrigée par le grattage du mot «saint». Alors que dans l'ensemble des lettres d'adhésion le projet ducal est jugé «bon, saint, juste et raisonnable », le correcteur de Chablis atrophie la portée de l'action de Jean sans Peur en la replaçant au strict plan humain. Il est significatif de voir, dans les lettres de Hesdin et leurs réponses, la prééminence des faits militaires s'estomper devant le prince accumulant les vertus civiles de justice et de paix. Dans ce cas c'est la signification spirituelle de l'entreprise qui est contestée. La multiplication des adhésions ne peut avoir la force d'un signe divin, "les propos saints" n'ont pas pour vocation de combattre une hérésie, simplement un adversaire politique. Plus grave, la correction introduit le vice et la suspicion quant au document comme sur les intentions de son auteur. Jean sans Peur recherche un pouvoir terrestre, corruptible, différent d'un monde sain où le loup repose à côté de l'agneau ${ }^{68}$. La gloire du duc de Bourgogne, sa doxa, n'est qu'apparence et repose sur la division du pouvoir et la promesse fallacieuse garantissant à tous un avenir de paix et de justice. Par leur nature, le contrôle et la correction du texte insultent le duc, en le prenant en mauvaise part.

\section{Réactiver la mémoire du serment}

A défaut d'un arbitrage, le rituel du serment apporte des symboles qui ravivent la mémoire collective et rappellent l'unité de la communauté. A Mâcon [fig.4] cette mémoire est ravivée par deux fois après l'assassinat de Montereau (1419). Lors de sa joyeuse entrée à Mâcon, en septembre 1424, Philippe le Bon choisit d'emprunter la porte du bourg Saveron ${ }^{69}$. La pauvreté et la composition sociale du quartier des artisans trouvent leur place dans la guerre des signes que se livrent les princes lors de la guerre civile. En 1417, les bourgeois ont affirmé leur fidélité aux propos de Jean sans Peur dont le programme de réforme s'exprime à travers l'image du rabot ${ }^{70}$. Cette image, positive et stable, s'oppose à ce qui est négatif, instable et déformé ${ }^{71}$. Avec l'instrument de l'artisan, Jean sans Peur s'adresse au petit peuple. En 1424, l'hommage des bourgeois à Philippe le Bon n'intervient qu'après cette démonstration de la permanence de la politique ducale.

En novembre 1435, l'hommage prêté à Philippe le Bon ravive la mémoire de saint Louis et du bon gouvernement de Jean sans Peur auquel est associée Marguerite de Bavière : « [le duc Jean et son épouse] désirant, de leur pouvoir, metre l'estat de France, lors sy désolé, en bon estat et debout, et hoster ceux qui estoient cause de le ainsi domagier et faire, de leur povoir, tenir ledit royaulme en l'estat que le noble nom de France le porte, et en l'estat et usaige que le noble roy saint Louis, fut roi de France, le tenoit, feirent recquerir les gens d'église, nobles et bourgeois des cité et comté de Mascon, qu'ils se voulsissent adhérer à leur bon et saint propos et tenir le parti de monseigneur le duc Jehan $[\ldots]^{72} \gg$. La cérémonie de 1435 réactive la mémoire de saint Louis qui marque la ville de son empreinte en 1255, celle du serment prêté et la fidélité jurée en 1417 à Jean sans Peur. Elle juxtapose dans la contemporanéité plusieurs fragments de temporalités différentes ${ }^{73}$. L'enjeu n'est plus d'asseoir une domination nouvelle mais d'assurer un contrôle sur la cité. Ce choix répond à trois logiques : valoriser la mémoire de la cité, affirmer la capacité des bourgeois à défendre leurs privilèges et montrer l'unité de la communauté civique.

Or le rituel reste une mise en scène. Il établit l'ordre du groupe dominant favorable à Jean sans Peur. Le rituel exclut volontairement l'étranger et le commun de Mâcon au profit des 
élites marchandes et lettrées du bourg commerçant. Le rituel du serment est bien un acte de violence. Le «serment novel» laisse peu de place pour l'opposition dont la capacité de nuisance, nous l'avons vu, reste efficace ${ }^{74}$. Il rétablit l'ordre par le groupe dominant et lui assure un meilleur contrôle social. Toutefois, les dissensions demeurent. Elles fissurent l'unité affichée artificiellement. D'ailleurs, les notables recommandent la ville à une tierce puissance: le duc Amédée VIII de Savoie. Il s'est à plusieurs reprises entremis dans les affaires de paix en proposant la neutralité de ses territoires pour des pourparlers ${ }^{75}$. En janvier 1423, son émissaire, Amé Macet, prend possession de la ville. Il détient des lettres d'Amédée VIII et deux copies, du duc de Bourgogne et de la duchesse de Bourbon ${ }^{76}$, dans lesquelles il est demandé que l'on obéisse au duc de Savoie. Cette tutelle prend fin en août avec la destruction des forces delphinales franco-écossaises à Cravant. Lors de la domination savoyarde sur la ville, les serments n'auront plus lieu chez les Dominicains mais dans la cathédrale Saint-Vincent. Le couvent des Dominicains participe à la guerre des signes que se livrent les princes car l'Ordre a la confiance de la maison de Bourgogne ${ }^{77}$. A Mâcon, le lieu du rituel est l'expression visuelle de la puissance partisane et le duc de Savoie cherche à s'en démarquer.

Le rituel du serment entre dans la stratégie communale du contrôle social, de l'emprise foncière et de la consolidation de la puissance de l'oligarchie urbaine. Les notables, urbains et ruraux, sans cesse menacés dans leur légitimité par les rivalités exacerbées lors de la guerre civile, recourent au «serment novel » pour fonder un pouvoir partisan. Un espace public s'affirme et triomphe à Mâcon comme à Charlieu, Vézelay ou Mailly-le-Château etc. Il donne naissance à un espace politique où s'expriment des tensions et des désaccords. Le cadre étroit de cet article ne permet que d'effleurer le sujet des rapports de force qui s'expriment au cours du rituel du serment. L'étendue du sujet et l'éparpillement des travaux justifient, dans le cadre de notre travail de doctorat, de poursuivre l'enquête.

Benoît Léthenet EA 3400 - UdS

\footnotetext{
${ }^{1}$ R. VERDIER (dir.), Le serment, Paris, éd. CNRS, 1991, 2 vol. ; F. LAURENT (dir.), Serment, promesse et engagement : rituels et modalités au Moyen Age, Presses Universitaires de la Méditerranée, 2008. I. ROSIERCATACH, La parole efficace : signe, rituel, sacré, Paris, Seuil, 2004 ; B. LEMESLE, « Le serment promis. Le serment judiciaire à partir de quelques documents angevins des XIe et XIIe siècles », in Crime, Histoire \& Sociétés / Crime, History \& Societies, vol. 6, 2, 2002, p. 5-28.

${ }^{2}$ N. OFFENSTADT, Faire la paix au Moyen Age, Paris, Perrin, 2007, p. 257-258.

${ }^{3}$ S. LECOINTRE, « Ma langue prêta serment », in R. VERDIER (dir.), op. cit., p. 5-22.

${ }^{4}$ H. KELLER, «"Kommune": Städtische Selbstregierung und mittelalterliche "Volksherrschaft" im Spiegel italienischer Wahlverfahren des 12. - 14. Jahrhunderts », in G. ALTHOFF (dir.), Person und Gemeinschaft im Mittelalter. Karl Schmidt zum 65. Geburtstag, 1988, p. 573-575.

F. BATTENBERG, «Dinggenossenschaftliche Wahlen im Mittelalter. Zur Wahl und Einsetzung von Schöffenkollegien und gerichtlichen Funktionsträgern, besonders vom 14. bis zum 16. Jahrhundert », in R. SCHNEIDER, H. ZIMMERMANN (dir.), Wahlen und Wählen im Mittelalter, Vorträge und Forschungen, Bd. 37, 1990, p. 274.

5 K. SCHULZ, «Wahlen und Formen der Mitbestimmung in der mittelalterlichen Stadt des 12.-13. Jahrhunderts », in R. SCHNEIDER, H. ZIMMERMANN (dir.), op. cit., p. 323.

${ }^{6}$ D. POECK, Rituale der Ratswahl. Zeichen und Zeremoniell der Ratssetzung in Europa, 12.-18. Jahrhundert, Köln, Böhlau, 2003.

${ }^{7}$ S. RÜTHER, « Soziale Distinktion und städtischer Konsens. Repräsentationsformen bürgerlicher Herrschaft in Lübeck », in M. FÜSSEL, Th. WELLER (dir.), Ordnung und Distinktion Praktiken sozialer Repräsentation in der ständischen Gesellschaft, 2005, p. 102-105.
} 
${ }^{8}$ N. OFFENSTADT, « Guerre civile et espace public à la fin du Moyen Age : la lutte des Armagnacs et des Bourguignons », in Guerres et extension de la participation politique, à paraître.

${ }^{9}$ Les notes critiques et les réflexions qui suivent doivent beaucoup aux journées d'études organisées par le LAMOP sur le thème: L'espace public au Moyen Age et en particuliers aux travaux introductifs de Patrick Boucheron «Espace public et lieux publics : approches en histoire urbaine », communication à la première journée du programme : L'espace public au Moyen Age, Paris I - LAMOP, 7 décembre 2004.

${ }^{10} \mathrm{~J}$.-Cl. PERROT, « Rapports sociaux et villes au XVIIIe siècle », in Annales ESC, 1968, p. 241-268.

${ }^{11}$ Pour les prémices d'un espace public au Moyen âge : B. THUM, «Öffentlichkeit und Kommunikation im Mittelalter. Zur Herstellung von Öffentlichkeit im Bezugsfeld elementarer Kommunikationsformen im 13. Jahrhundert » in Höfische Repräsentation. Das Zeremoniell und die Zeichen, Tübingen, 1990, p. 65-87 ; N. OFFENSTADT, «Les crieurs publics à la fin du Moyen âge. Enjeux d'une recherche », in Information et société en Occident à la fin du Moyen Âge (Colloque, Montréal et Ottawa, 2002), Paris, 2004, p. 203-217 ; N. OFFENSTADT, "'L'histoire politique' de la fin du Moyen Âge. Quelques discussions », in Etre historien du Moyen Age au XXIe siècle, Paris, Publications de la Sorbonne, 2008, p. 179-198.

${ }^{12}$ C. GAUVARD «Qu'est-ce que l'opinion avant l'invention de l'imprimerie ? », in L'Opinion. Information, rumeur, propagande (Les Rendez-vous de l'Histoire, Blois, 2007), s.l., Editions Pleins feux, 2008, p. 21-59; B. GUENEE, L'opinion publique à la fin du Moyen Age, Paris, Perrin, 2002 ; pour une critique épistémologique, S. HABER, «Quelques mots pour historiciser L'espace public de Habermas » communication dans le cadre du programme : L'espace public au Moyen Age, Paris I - LAMOP.

${ }^{13}$ T. PAQUOT, L'espace public, Paris, La découverte, 2009.

${ }^{14}$ A. RIGAUDIERE, Penser et construire l'Etat dans la France au Moyen Age (XIIIe-XVe siècle), Paris, Comité pour l'histoire économique et financière de la France, 2003, p. 320.

15 E. CROUZET-PAVAN, « «Pour le bien commun». A propos des politiques urbaines dans l'Italie communale », in Pouvoir et édilité. Les grands chantiers dans l'Italie communale et seigneuriale, Rome, Ecole Française de Rome, 2003 (collection de l'EFR, 302), p. 11-40, cité dans : P. BOUCHERON, «Espace public et lieux publics : approches en histoire urbaine », communication à la première journée du programme : L'espace public au Moyen Age, Paris I - LAMOP, 7 décembre 2004.

16 J. HEERS, Espaces publics, espaces privés dans la ville. Le Liber terminorum de Bologne (1294), Paris, CNRS, 1984 ; B. VINCENT, « Espace public et espace privé dans les villes andalouses (XVe-XVIe siècles), in J.-Cl. MAIRE VIGUEUR (éd.), D'une ville à l'autre : structures matérielles et organisation de l'espace dans les villes européennes (XIIIe-XVIe siècles). Actes du colloque organisé par l'Ecole Française de Rome (Rome, $I^{\text {er }}$ 4 décembre 1986), Rome, 1989, p. 711-724; E. VAVRA (éd.), Virtuelle Raüme. Raumwahrnehmung und Raumvorstellung im Mittelalter, Berlin, Akademie Verlage, 2005 ; K. OSCHEMA, «Espaces publics autour d'une société de cour : l'exemple de la Bourgogne des ducs Valois », communication lors de la journée du programme : L'espace public au Moyen Age, Paris I - LAMOP, 5 décembre 2006.

${ }^{17}$ P. BOUCHERON, Villes d'Italie, Paris, Belin, 2004.

${ }^{18}$ E. LECUPPRE-DESJARDIN, La ville des cérémonies. Essai sur la communication dans les anciens Pays-Bas bourguignons, Turnhout, Brepols (« Studies in European Urban History », 4), 2004.

${ }^{19}$ J. BLANCHARD, «Le spectacle du rite : les entrées royales », in Revue historique, 627, 2003, p. 475-519.

${ }^{20}$ M. CAMILLE, «Signs of the city: place, power and public fantasy in medieval Paris », in B. A. HANAWALT, M. KOBIALKA (éd.), Medieval Practices of Space, Minneapolis, University of Minnesota Press, 2000, p. 1-36 et «Signs on Medieval Street Corners», in G. JARITZ (dir.), Die Strasse : zur Funktion und Perzeption öffentlichen Raums im späten Mittelalter, Vienne, 2001.

${ }^{21}$ J.-Cl. PERROT, « Rapports sociaux... », in op. cit., p. 241-268.

${ }^{22}$ D. ROUSSEL, Sur la place publique: sociabilités populaires et conflits à Paris au XVIe siècle, thèse de doctorat en cours, sous la direction de Robert Muchembled, Université Paris XIII - CRESC ; V. CHALLET, Mundere et auffere erbas. La révolte des Tuchins en Languedoc (1381-1384), Thèse dactylographiée, 2003, 3 vol. ; C. GAUVARD, «Les révoltes du règne de Charles VI : tentative pour expliquer un échec ", in Révolte et société (Colloque, Paris, 1988), Paris, 1989, p. 53-61. Les modernistes aboutissent aux mêmes conclusions : E. Le ROY LADURIE, Le Carnaval de Romans. De la chandeleur au mercredi des Cendres 1579-1580, Paris, 1989 ; Y.-M. BERCE, Fête et révolte. Des mentalités populaires du XVIe au XVIIIe siècle, Paris.

${ }^{23}$ M. DETIENNE, Qui veut prendre la parole ? (Le genre Humain, 40-41), 2003.

${ }^{24}$ E. PERARD (éd.), Recueil de plusieurs pièces curieuses servant à l'histoire de la Bourgogne, Paris, 1664.

${ }^{25}$ Dom PLANCHER, Histoire générale et particulière de Bourgogne, Dijon, 1739-1781, III, preuves : folios ccciij-cccvj.

${ }^{26}$ BARANTE, Histoire des ducs de Bourgogne de la Maison de Valois, 1364-1477, IV, Paris, 1826, p. 285-294.

${ }^{27}$ E. FOURNIAL, Charlieu. Des origines à l'aurore des temps modernes, Saint-Etienne, 1985, p. 253; A. CHEREST, Étude historique sur Vézelay, dans : Bulletin de la Société des sciences historiques et naturelles de l'Yonne, 1868, 22 ${ }^{\mathrm{e}}$ année, pièces justificatives, p. 612-613. 
${ }^{28}$ Collection de Bourgogne, LV, Paris, Bibliothèque Nationale, f. $257 \mathrm{r}^{\circ}$; Abbé LEBEUF, Mémoires concernant l'histoire civile et ecclésiastique d'Auxerre et de son ancien diocèse, t.4, nº349, p. 238.

${ }^{29}$ M. CANAT, Documents inédits pour servir à l'histoire de la Bourgogne, I, Chalon, 1863, p. XVIII : chaque document édité permet d'entrevoir « la consécration légale de certains droits politiques, dont chacun marque une étape dans les avancements de la liberté locale »

${ }^{30}$ Nous nous limitons, dans la bibliographie très importante sur la Bourgogne, à ne citer que quelques exemples : B.-A. POCQUET DU HAUT-JUSSE, La France gouvernée par Jean sans Peur, Paris, PUF, 1959 ; J. RICHARD, "Trois lettres concernant l'occupation de Mâcon par les Bourguignons (1417) », in Annales de Bourgogne, 33, 1961, p. 88-98 ; R. VAUGHAN, John the Fearless : The growth of burgundian power, Londres, 1966, p. 216 ; B. SCHNERB, Jean sans Peur. Le prince meurtrier, Paris, Payot, 2005.

${ }^{31}$ Notre travail de thèse porte sur le thème de la circulation de l'information, à Mâcon principalement, durant la guerre de Cent ans (1386-1435). La soutenance est prévue au printemps prochain à l'UdS.

${ }_{32}$ B. GUENEE, La folie de Charles VI, roi bien aimé, Paris, Perrin, 2004.

${ }^{33}$ Le traité d'Alliance fut signé le 15 avril 1410 marquant la naissance du parti antibourguignon et pour renforcer leurs liens, les conjurés décidèrent du mariage de Bonne d'Armagnac avec Charles d'Orléans alors âgé de 16 ans.

${ }^{34}$ B. SCHNERB, L’Etat Bourguignon, Paris, Perrin, 1999 (rééd. 2005).

${ }^{35}$ G. Le BOUVIER, dit Héraut Berry, Les Chroniques du roi Charles VII, H. COURTEAULT, L. CELIER (éd.), Paris, Klincksieck, société de l'Histoire de France, 1979.p. 74.

${ }^{36}$ Le Bouvier, Gilles, dit Héraut Berry, op. cit., p.77.

${ }^{37}$ Mémoires de Pierre de Fénin, escuyer et panetier de Charles VI (1407-1422), Buchon, A. (éd.), Paris, p.280.

${ }^{38}$ B. LÉTHENET, «Le «nouvel seigneur de la Conté de Mascon». La prise de contrôle de Mâcon par la Bourgogne (1417-1435) », in Annales de Bourgogne, 80, 2008, p. 39-100.

${ }^{39}$ AMM, Affaires militaires, EE41/35 ; ADCO, B11895/40.

${ }^{40}$ ADCO, B11895/39.

${ }^{41}$ N. OFFENSTADT, «Le roi de paix pendant la Guerre de Cent ans », in S. CAUCANAS, R. CAZALS, N. OFFENSADT (dir.), Paroles de paix en temps de guerre, Toulouse, Privat, 2006, p. 255-267.

${ }^{42}$ ADCO, B11895/40.

${ }^{43}$ M. MOLLAT, (éd.), Comptes généraux de l'Etat bourguignon de 1416 à 1420, Paris, Imprimerie

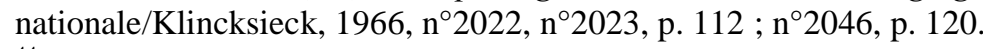

${ }^{44}$ ADCO, B11895.

${ }^{45}$ Des bourgeois aux citoyens : les lettres de serments de la ville de Strasbourg, livret de l'exposition présentée aux Archives de la Ville et de la Communauté urbaine de Strasbourg, du 25 février au 27 juin 2008.

${ }^{46} \mathrm{AMM}$, Administration communale, BB13, f. $1 \mathrm{v}^{\circ}$, f. $6 \mathrm{r}^{\circ}$, f. $7 \mathrm{v}^{\circ}$; BB15, f. $20 \mathrm{v}^{\circ}$.

${ }^{47}$ M. CANAT (éd.), Documents pour servir à l'histoire de la Bourgogne, I, Chalon, 1881, p. 335-336.

${ }^{48}$ AMM, Administration communale, BB12, f. $39 \mathrm{r}^{\circ}-\mathrm{v}^{\circ}$.

${ }^{49}$ ADCO, B11895/40, lettres de Marcigny.

50 Vidimus mâconnais des lettres de Hesdin vidimées à Dijon, du 31 janvier 1418 [n.s.], collationné par Olivier Berthoud tabellion de la prévôté de Mâcon. Mâcon, archives municipales, EE41/30.

${ }^{51}$ Vidimus dijonnais du 11 septembre 1417, parchemin, sceau de cire rouge sur double queue de parchemin, collationné au document côté B11895/39 par Oudot le Bediet et Jacot Boison coadjuteurs du tabellion Philippe Musnier. Mâcon, Archives municipales, EE41/31.

${ }^{52}$ Une étude sur la série des bulletins du vote préliminaires à la soustraction d'obédience a été menée par $\mathrm{H}$. MILLET, E. POULLE, Le vote de la soustraction d'obédience de 1398, Paris, Ed. du Centre national de la recherche scientifique, 1988.

${ }^{53}$ ADCO, B 11895/39bis

${ }^{54}$ Nous renvoyons aux propos de J. MORSEL, «Ce qu'écrire veut dire au Moyen Âge... Observations préliminaires à une étude de la scripturalité médiévale » in Memini. Travaux et documents de la Société des études médiévales du Québec, 4, 2000, p. 3-43: «c'est donc par rapport à ce qui ne circule pas et par rapport à ce qui n'est pas conservé que joue l'efficacité performative de l'écriture ».

${ }^{55}$ P. CAMMAROSANO, Le forme della propaganda politica nel Due e nel Trecento, Rome, Ecole française de Rome, 1994 ; M. CLANCHY, England and its rulers, 1066-1272: foreign lordship and national identity, Londres, Fontana press, 1983.

${ }^{56}$ AMM, Administration communale, BB12, f. $38 v^{\circ}$.

${ }^{57}$ T. DUTOUR, «Élaboration, publication, diffusion de l'information à la fin du Moyen Âge (Bourgogne ducale et France royale », in D. LETT, N. OFFENSTADT (dir.), Haro! Noël! Oyé! Pratiques du cri au Moyen Âge, Publications de la Sorbonne, Paris, 2003, p. 140-155.

${ }^{58}$ AMM, Administration communale, BB13, f. $81 v^{\circ}$; BB15, f. $54 v^{\circ}$. 
${ }^{59}$ F. MENANT, J.-P. JESSENNE (éd.), Les élites rurales dans l'Europe médiévale et moderne, Actes des XXVIIe journées internationales d'Histoire de l'Abbaye de Flaran, Toulouse, PU du Mirail, 2007.

${ }^{60}$ AMM, Administration communale, BB12, f. $94 v^{\circ}$.

${ }^{61}$ Guy de La Trémoille, seigneur de Bourbon-Lancy et d'Uchon, comte de Joigny (attesté de 1410-v.1438).

${ }^{62}$ ADCO B11895/40 lettres de Tournus : « Nous, prieur et couvent du royal monastére de Tornus reverend pere en Dieu monseigneur l'abbé dudit monastére estant ou saint concile de Constance »; AMM EE41/35 : « reverend pere en Dieu monseigneur Jehan, par la grace de Dieu evesque de Mascon estant a present absent du pais ».

${ }^{63}$ ADCO B11895/40 lettres de Chaumont, le lieutenant du bailli fait certifier les lettres d'adhésion auprès du bailli de Langres.

${ }^{64}$ C'est le cas de Chablis, Sacy, Charny, Villiers-Saint-Benoît, Vézelay, Mailly-le-Château et Saint-Bris. Les seigneurs laïcs sont les suscripteurs à Chêne Arnoult, Villefranche et Charny. La prévôté valide les actes à Brinon, Vermenton et Saint-Bris.

${ }^{65}$ AMM, Administration communale, BB14 f. 12r ${ }^{\circ}$; AMM, Comptes de la commune, CC66/48, CC70/54.

${ }^{66}$ On sait par ailleurs, que pour parer au ralliement de Mailly-le-Château, le dauphin Charles offre l'année suivante (1418) les reliques de saint Adrien à l'église du lieu. Est-ce un acte de dévotion ou un message politique ? Le supplice à coups de bâton n'est pas sans rappeler le bâton noueux des Orléans. L'avertissement est clair pour les rebelles à son autorité. En revanche, Adrien professant la foi chrétienne et rejoignant volontairement les martyrs du Christ est un exemple qui plaide en faveur du ralliement au dauphin, cité dans : N.-L. MARTIN, Précis historique et anecdotes diverses sur la ville et l'ancienne abbaye de Vézelay et ses environs, Auxerre, 1862, p. 182.

${ }^{67}$ N.-L. MARTIN, op. cit., p. 180-181.

${ }^{68}$ Is, $11,6$.

${ }^{69}$ Archives municipales de Mâcon, Administration communale, BB14, f. 17r ${ }^{\circ}$.

${ }^{70}$ S. SLANIČKA, Krieg der Zeichen. Die visuelle Politik Johanns ohne Furcht und der armagnakischburgundische Bürgerkrieg, Göttingen, Veröffentlichungen des Max-Planck-Instituts für Geschichte, Band 182, 2002.

${ }^{71}$ JEAN MOLINET, Chroniques, J.A.C. BUCHON (éd.), Paris, 1827, p.10, contre le bâton noueux d'Orléans.

${ }^{72}$ M. CANAT (éd.), op. cit., p. 264-266.

${ }^{73}$ B. LEPETIT, «Une herméneutique urbaine est-elle possible ?», in B. LEPETIT et D. PUMAIN (dir.), Temporalités urbaines, Paris, Anthropos, 1993, p. 287-299.

${ }^{74}$ M. CANAT (éd.), op. cit., p. 307 ; p. 317 ; p. 332.

${ }^{75} \mathrm{AMM}$, Administration communale, BB13, f. $55 \mathrm{v}^{\circ}$, f. $70 \mathrm{v}^{\circ}$, f. $74 \mathrm{r}^{\circ}-\mathrm{v}^{\circ}$, f. $79 \mathrm{v}^{\circ}$, f. $80 \mathrm{v}^{\circ}$-f. $81 \mathrm{v}^{\circ}$.

${ }^{76}$ Ibid., BB13, f. $80 v^{\circ}$.

${ }^{77} \mathrm{~V}$. TABBAGH, «Un siècle d'influence à la cour des ducs Valois de Bourgogne », in Annales de Bourgogne, 80, 2008, p. 101-124. 